\title{
Pigeons May Not Use Dual Coding in the Radial Maze Analog Task
}

\author{
Kelly A. DiGian and Thomas R. Zentall \\ University of Kentucky
}

\begin{abstract}
Using a radial maze analog task, T. R. Zentall, J. N. Steirn, and P. Jackson-Smith (1990) found evidence that when a delay was interpolated early in a trial, pigeons coded locations retrospectively, but when the delay was interpolated late in the trial, they coded locations prospectively (support for a dual coding hypothesis). In Experiment 1 of the present study, the authors replicated the original finding of dual coding. In Experiments 2 and 3, they used a 2-alternative test procedure that does not require the assumption that pigeons' choice criterion, which changes over the course of the trial, is the same on delay and control trials. Under these conditions, the pigeons no longer showed evidence for dual coding. Instead, there was some evidence that they showed prospective coding, but a more parsimonious account of the results may be that the delay produced a relatively constant decrement in performance at all points of delay interpolation. The original finding of dual coding by Zentall et al. might have been biased by more impulsive choices early in control trials but not in delay trials and by a more stringent choice criterion late in delay trials.
\end{abstract}

Keywords: radial maze analog, pigeons, dual coding, prospective coding, retrospective coding

In delayed matching to sample, choice of one test or comparison stimulus is correct following the presentation of one sample, whereas choice of the other comparison stimulus is correct following the presentation of the other sample. If a delay is inserted between the offset of the sample and the onset of the comparison stimuli, memory processes can be examined. One possibility is that the memory code may consist of a representation of the sample (i.e., the memory code may be retrospective in nature). Alternatively, the memory code may consist of a representation of the correct comparison or a response intention (i.e., the memory code may be prospective in nature). Although most of the evidence suggests that animals use retrospective codes, there is evidence that both retrospective and prospective codes can be used by pigeons (Grant, 1993; Honig, 1978, 1981; Honig \& Thompson, 1982; Roitblat, 1993; Steirn, Zentall, \& Sherburne, 1992; Wasserman, 1986; Zentall, 1998; Zentall, Steirn, \& Jackson-Smith, 1990) and rats (Cook, Brown, \& Riley, 1985).

\section{Evidence for Flexible Within-Trial Coding Processes}

Under some conditions, it appears that animals may use flexible coding strategies involving retrospective or prospective coding according to the task demands. Although there is some evidence for such flexible coding in matching-to-sample procedures (e.g., Zentall, Urcuioli, Jagielo, \& Jackson-Smith, 1989), much of the evidence for flexible coding comes from experiments using the radial maze procedure, in which each of several alternatives must be chosen once and only once (Cook et al., 1985; Zentall et al., 1990). Cook et al. (1985), for example, found evidence for within-

Kelly A. DiGian and Thomas R. Zentall, Department of Psychology, University of Kentucky.

Correspondence concerning this article should be addressed to Thomas R. Zentall, Department of Psychology, University of Kentucky, Lexington, KY 40506-0044. E-mail: zentall@uky.edu trial flexible coding by rats. Cook et al. used a radial maze in which 12 baited arms radiated out from a central platform. The rats were placed on the central platform and were allowed to make arm choices to retrieve a small amount of food placed at the end of each arm. Cook et al. analyzed the pattern of errors made (revisits to arms already visited) resulting from the insertion of a delay at a point in the trial that varied from trial to trial, to make inferences about the coding strategy that rats used as they progressed through the trial. After a 15-min delay was inserted following 2, 4, 6, 8, or 10 correct choices, the rats were allowed to complete the trial. To compare the likelihood of making errors at the different points of delay interpolation (PDIs), the authors corrected errors for opportunity, because the probability of making an error by chance increases as more correct choices have been made.

Cook et al. (1985) noted that a second correction was needed because as the probability of making an error by chance increased, the criterion for making a choice appeared to change for their rats as they progressed through the trial. On control trials, without a delay, Cook et al. found that the rats tended to make proportionally more errors (relative to chance) early in the trial than they did late in the trial. This change in proportional errors made was attributed to the fact that absolute errors were more likely to occur late in the trial than early in the trial and that the rats appeared to compensate for that fact by choosing arms more carefully later in the trial. To control for this change in error rate not attributable to the delay, Cook et al. subtracted control errors from delay errors at each PDI (control trial errors were calculated at each PDI as if a delay had occurred-a pseudodelay).

To examine the coding strategy used by the rats, Cook et al. (1985) compared the adjusted error rate as a function of the PDI. They reasoned that the greater the memory load was, the greater should be the disruptive effects of the delay. Thus, on the radial maze, a retrospective coding strategy would result in more adjusted errors later in the trial because memory load should increase as more arms are chosen. However, a prospective coding strategy 
would create more adjusted errors early in the trial because memory load should decrease as arms are chosen.

These strategies also make different predictions about the serial position functions for errors. The serial position functions represent the relationship between the order of arm choices before the delay and the tendency for the rats to revisit those arms. If there were a primacy effect, the rats should remember items at the beginning of the predelay list better than items at the end of the list, whereas if there were a recency effect, rats would remember items at the end of the list better than items at the beginning of the list. Thus, if the rats were retrospectively coding choices made, the serial position of the arms already visited should affect the likelihood of making an error. However, if the rats were prospectively coding choices not yet made, the pattern of errors should not be influenced by the order in which the arms were visited prior to the delay, because the previously visited arms are presumably not represented in memory.

The results of Cook et al.'s (1985) Experiment 1 revealed an increase in errors as the PDIs increased from 2 to 4 to 6 and a decrease in errors as the PDIs further increased from 6 to 8 to 10 . These results suggest that their rats were using a dual coding strategy. That is, they coded choices already made retrospectively if the delay occurred early in the trial, but they coded choices yet to be made prospectively if the delay occurred later in the trial. The serial position curves also supported a dual coding account. The data from early PDIs (4 and 6) showed a recency effect, but data from late PDIs ( 8 and 10) showed neither a recency effect nor a primacy effect. These serial position functions are consistent with a retrospective coding account early in the trial and a prospective coding account late in the trial.

Cook et al. (1985) recognized that it was possible that their assumption about the adjustment for errors not attributable to the delay was not correct. That is, because the choice criterion changed as the rat progressed through the trial, it might not have been correct to assume that subtracting control (nondelay) errors from delay errors at each PDI would leave only errors attributable to the delay. Cook et al. reasoned that if their rats were more careful in making their choices following a delay than on trials without a delay and if that tendency increased late in a trial, it was possible that their control trial adjustment overcorrected and merely gave the illusion of late-PDI prospective coding.

To avoid the need to adjust the errors for opportunity so that errors could be compared among the PDIs, the authors used a two-alternative forced-choice procedure (Cook et al., 1985, Experiment 4). That is, following a delay, the rats were forced to choose between one previously visited arm and one previously unvisited arm. This procedure makes the probability of choosing the correct arm by chance the same (50\%) at all PDIs, and performance at the different PDIs can be compared directly. The results found with this forced-choice procedure were consistent with the results of the earlier procedure, in which the rats had to complete the trial following a delay. That is, the results were consistent with a dual coding account. Furthermore, once again, serial position curves supported a retrospective coding account at PDIs 4 and 6 and a prospective coding account at PDIs 8 and 10. Thus, the results from Cook et al.'s experiments with rats in the radial maze supported a dual coding account.

To test the generality of the flexible coding strategy used by rats, Kesner and DeSpain (1988) tested human subjects with lists that consisted of 2, 4, 6, 8, 10,12, or $14 X$ s. Each $X$ was presented alone in a specific location on a grid of 16 squares. After a short delay, Kesner and DeSpain tested subjects by presenting one $X$ that had been presented in the list on that trial and one $X$ that had not been presented on that trial. Subjects were told to choose the $X$ that had not been presented on that trial. Kesner and DeSpain found individual differences in strategy for performing the task. They found that some subjects used a pure retrospective coding strategy. For these subjects, there was an increase in errors with increasing PDI. However, other subjects appeared to use a dual coding strategy. These subjects made an increasing number of errors as the number of $X \mathrm{~s}$ increased from 2 to 8 , but they made a decreasing number of errors as the number of $X$ s increased further from 8 to 14 . These results suggest that some subjects remembered the list of $X \mathrm{~s}$ that had been presented on each trial, whereas others remembered the set of fewer items, $X \mathrm{~s}$ that had been presented at early PDIs and empty grid spaces at later PDIs.

Why some human subjects used a pure retrospective coding strategy and others used a dual coding strategy is not clear, but it may be related to past experience and the small number of training trials used in the research with humans. Alternatively, it may be that rats' use of a dual coding strategy is related to their natural propensity to acquire spatial problems, and on a radial maze they tend to visit each arm without revisits even when no food is present (Hoffman, Timberlake, Leffel, \& Gont, 1999).

As a further test of the generality of the dual coding hypothesis, Zentall et al. (1990) asked whether pigeons, which do not have such a natural tendency to explore all of the alternatives, would show evidence of using a dual coding strategy when trained on a radial maze analog task. Their display consisted of five response keys, and, as on the radial maze, the first response to any key was rewarded, but additional responses to the same key were not. Each trial continued until all five keys had been chosen. In Experiment 1 , although the pigeons initially showed a strong bias to make revisits, they did learn to avoid revisits to the response keys. Once a high level of performance was attained, on selected trials a delay was inserted after the pigeon made one, two, three, or four correct choices. When the pattern of adjusted errors was examined at each PDI, the pigeons were found to perform better early and late in the trial (i.e., they performed better at PDIs 1 and 4 than at PDIs 2 and 3 ), a pattern consistent with the dual coding strategy found for rats (see also Steirn et al., 1992).

In the procedure used by Zentall et al. (1990), following the delay, the pigeons were required to complete the trial by selecting all of the response keys not already selected prior to the delay. As noted earlier, however, the adjustment in errors used to correct for opportunity among the PDIs requires an assumption about the comparability of control and delay errors not attributable to memory loss produced by the delay. When Cook et al. (1985) used a more direct two-alternative test that did not require such an assumption, they still found evidence for dual coding by rats; however, we thought that it would be wise to conduct a similar unbiased, two-alternative test with pigeons.

The purpose of the present experiments was to first replicate the results of Zentall et al. (1990) using fixed delays of $15 \mathrm{~s}$ and $30 \mathrm{~s}$ and then test for the presence of dual coding using a twoalternative forced-choice procedure that does not require correction for the opportunity to make errors, as is the case when five keys are available after the delay. In the forced-choice procedure, 
two response keys are presented after the delay. One alternative is correct (was not chosen before the delay), and one alternative is incorrect (was chosen before the delay). With this procedure, the probability of making an error by chance is the same at all PDIs: $50 \%$. In addition, one can include control trials, with forced choices but no delay, to detect any systematic changes in errors over the course of the trial that might not be attributed to the delay. This procedure provides a test of the hypothesis that the results obtained by Zentall et al. (1990) might have been attributable to differences in choice criterion resulting from the changing probability of making an error by chance with increasing PDI.

According to the dual coding hypothesis, pigeons should do better at PDIs 1 and 4 compared with PDIs 2 and 3. Conversely, if subtracting relative control errors from delay errors, as Zentall et al. (1990) did, provides a biased estimate of errors attributable to the delay, evidence for dual coding may not be found

\section{Experiment 1}

\section{Method}

\section{Subjects}

Six unsexed White Carneaux pigeons (Columba livia) were used as subjects. They were purchased as retired breeders from the Palmetto Pigeon Plant (Sumter, SC). The pigeons were free fed until their weight had stabilized and were then put on a restricted diet to reduce them to $85 \%$ of their free-feeding weight. This weight was maintained throughout the experiment. The pigeons were housed with free access to water and grit in a colony room that was placed on a 12-hr light-dark cycle.

\section{Apparatus}

A standard operant chamber (BRS/LVE, Beltsville, MD) was used. The operant chamber was $35 \mathrm{~cm}$ high, $30 \mathrm{~cm}$ wide, and 35 $\mathrm{cm}$ across the response panel. A houselight was located in the ceiling $13 \mathrm{~cm}$ from the response panel and $16 \mathrm{~cm}$ from the door of the chamber. There were 25 keys on the response panel, which were arranged in a $5 \times 5$ matrix. Five keys were used during the experiment, the four corner keys and the key in the center of the matrix. Each key was $1.6 \mathrm{~cm}$ in diameter, with $3.1 \mathrm{~cm}$ separating the centers of adjacent keys. Each key was illuminated by one hue (blue, top left; red, top right; green, bottom left; yellow, bottom right; and white, center) consistently throughout the experiment. Thus, the hue and the spatial location provided redundant cues (see Zentall et al., 1990, for a rationale for this redundancy). A feeder provided mixed grain through an opening $5.7 \mathrm{~cm}$ high and $5.1 \mathrm{~cm}$ wide. The feeder opening was located $6 \mathrm{~cm}$ above the floor of the chamber.

\section{Procedure}

Pretraining. During the first phase of pretraining, one response key was illuminated at a time. The pigeons were trained to peck the illuminated key and were reinforced with $1.5 \mathrm{~s}$ of access to mixed grain (the type and duration of reinforcement used throughout the experiment). They were required to peck the illuminated key one time for reinforcement, with a 10-s intertrial interval during which the houselight was illuminated. Once they completed four sessions of 50 trials per day, they began the next phase of pretraining. The pigeons were trained 6 days a week throughout the experiment.

During the second phase of pretraining, all five keys were illuminated at the beginning of each trial. The first response to any key was reinforced. Keys were not lit during the reinforcement interval. The chosen key remained dark after reinforcement, but keys that had not been chosen were reilluminated. Any response to a remaining key turned off that key and provided access to reinforcement. This process continued until each of the five keys had been pecked. During this second phase of pretraining, trials were separated by a 60 -s lit intertrial interval. There were two of these sessions of 24 trials per day. Then the response requirement was increased to three pecks for two sessions and finally to five pecks for two more sessions. After completing pretraining, the pigeons began the training phase.

Training. The first phase of training was similar to the last phase of pretraining in that all five keys were illuminated at the beginning of each trial. Five pecks to any key was considered a choice, and initial choices to each key produced access to reinforcement, but unlike pretraining, that chosen key was turned back on after reinforcement. Because all five keys were reilluminated after the reinforcement interval, during this phase of training, the pigeon was able to choose keys that it had chosen earlier in the trial. These choices were considered errors and resulted in the offset of all keys for $2.5 \mathrm{~s}$. The trial ended when all five keys had been chosen at least once. Trials were separated by a 60-s intertrial interval during which the houselight was illuminated. The pigeons completed 15 trials per day, and they were trained until they completed three consecutive sessions with an average of fewer than three errors per trial.

Delay testing. After reaching criterion in the training phase, the pigeons began testing trials with delays. There were 10 trials in each delay session. On 2 control trials, just as in training, pigeons were able to make choices until they had chosen all five keys at least once. The other 8 trials began with all five keys illuminated and included a delay. The point in the trial at which the delay was inserted, the PDI, was randomly chosen by the computer program, with the constraint that there were 2 trials for each PDI (i.e., after one, two, three, or four reinforced choices). On these trials, pigeons made choices according to the contingencies established in training until the delay began. The delay began after the last correct choice for that particular PDI and consisted of $15 \mathrm{~s}$ of darkness. After the delay, all keys were reilluminated, and the trial continued until the pigeon had chosen all keys that it had not chosen before the delay. Reinforcement was provided for all initial choices, and $2.5 \mathrm{~s}$ of darkness followed all incorrect choices, as in training. There was one block of 10 sessions with a 15-s delay, and then pigeons began testing sessions with a 30 -s delay. All pigeons completed nine blocks of 10 sessions with a 30 -s delay.

\section{Results}

\section{Training}

Pigeons made about nine errors per trial during the first block of 10 sessions. Errors decreased to about four errors per trial by the third block of 10 sessions and leveled off at just below three errors per trial by Block 7 . The mean number of sessions to reach criterion was $34.2($ SEM $=8.36)$. 


\section{Delay Testing}

Overall, pigeons made an average of about 2.5 errors on control trials and about 3.0 errors on delay trials. A Block $\times$ Trial Type (control vs. delay) analysis of variance (ANOVA) was performed with the error data. A significant effect of trial type was obtained, $F(1,5)=43.61, p<.05$, but no significant effects of block or the Block $\times$ Trial Type interaction were found, $F(9,45)=1.74, p>$ .05 , and $F<1$, respectively.

Errors were analyzed further to determine whether dual coding was used on delay trials. First, the number of errors attributable to the delay was calculated. Errors attributable to the delay are choices made after the delay to response keys that had been chosen before the delay. These errors were summed for each PDI. This total was divided by the number of possible errors that could have been made for that PDI. For example, on PDI 2 trials, two correct choices were made before the delay, so there were two possible ways to make an error after the delay. If the pigeon made one of these errors, the proportion of errors at that PDI would have been .5. If more than one error was made to a particular location after the delay, only the first error was counted.

Errors were also corrected for the potential change in choice criterion as the trial progressed. This correction was obtained for each pigeon via errors made on control trials at each point in the trial following a reinforcement. A measure of control errors was calculated as if a delay had occurred at that point in the trial. We refer to these errors that are not attributable to the delay as pseudodelay errors. For each control trial, we calculated the number of errors for each point in the trial following a reinforcement by counting the number of errors made after the pseudodelay to keys that had been chosen before that point. For example, we analyzed the sequence of choices on a control trial by examining choices made following two reinforced choices (the pseudodelay) for errors made to the location of either of the first two correct choices. According to this method, the number of opportunities to make an error equals the number of correct choices prior to the pseudodelay. We then divided the number of errors made after the pseudodelay to locations prior to the pseudodelay by the number of opportunities to make an error (in this case, 2). These calculations, pooled over the last 50 sessions of testing, are shown in Figure 1 for control trials and delay trials.

As can be seen in Figure 1, the proportion of control errors decreased with increasing PDI. This decrease can be attributed to the changing criterion for making a choice at later PDIs. The proportion of delay errors also decreased with increasing PDI; however, it first increased from PDI 1 to PDI 2.

\section{Serial Position Effects}

When the adjusted errors on control trials were subtracted from the adjusted errors on delay trials, the resulting error function represented the proportion of errors attributable to the delay. These proportions, at each PDI, are presented in Figure 2. As can be seen in Figure 2, the errors attributable to the delay increased from PDI 1 to PDI 2 and then decreased from PDI 3 to PDI 4. Initial analyses tested whether adjusted errors first increased and then decreased. These tests revealed significant differences between PDIs 1 and 2, $t(5)=3.33, p<.05$, and PDIs 3 and $4, t(5)=4.85, p<.05$. A repeated measures ANOVA was conducted with PDI as the factor.

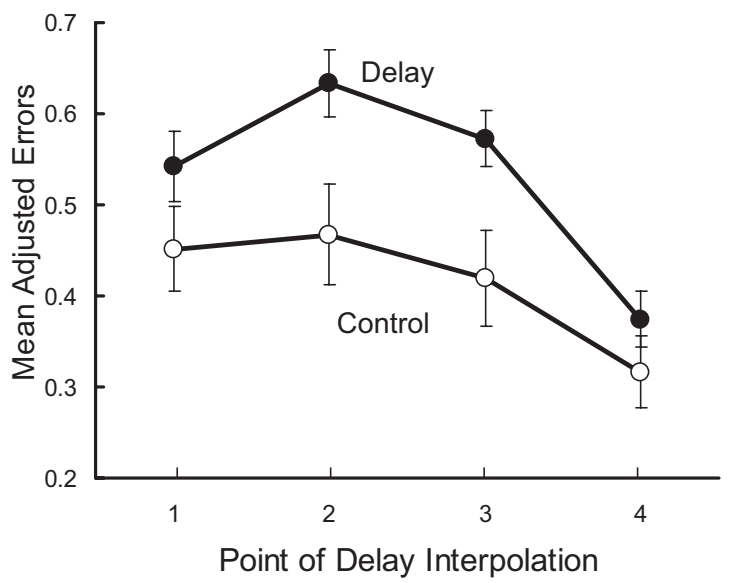

Figure 1. Mean adjusted control and delay errors in Experiment 1 (error bars represent standard error of the mean) as a function of the point of delay interpolation.

The ANOVA indicated that there was a significant main effect of PDI, $F(3,15)=5.05, p<.05$. The errors attributable to the delay function were also analyzed for linear and quadratic components. The analysis indicated that there was no significant linear trend $(F<1)$ but that there was a significant quadratic trend, $F(1,5)=$ $30.48, p<.05$. The significant quadratic trend resulted from the inverted $U$-shaped function (shown in Figure 2), which is consistent with the dual coding hypothesis.

We examined serial position effects by calculating errors to each serial position for each PDI as a proportion of the total possible errors that could have been made at that position. For example, on PDI 2 trials, errors could be made to the key chosen first or the key chosen second. Errors to the first choice were counted as errors to Serial Position 1, and errors to the second choice were counted as errors to Serial Position 2. The proportion of errors at each position at each PDI was calculated for control trials and delay trials separately. Control trial errors were subtracted from delay trial errors, and the difference scores at each position were compared with a $t$ test for PDI 2 and ANOVAs for PDIs 3 and 4. The adjusted errors at each serial position are shown in Figure 3. At PDI 2, errors appeared to increase from Position 1 to Position 2, but this increase was not statistically significant, $t(5)=2.50, p>.05$. Similarly, at PDI 3, there appeared to be an increase in errors from Positions 1 to 3 , but again there was no significant effect of serial position, $F(2,10)=0.73, p>.05$. Finally, the ANOVA using errors at Positions 1 to 4 on PDI 4 trials revealed no significant effect of serial position, $F(3,15)=1.90, p>.05$. Although significant serial position errors were not found, consistent with Zentall et al. (1990), there was a tendency for errors to increase with increasing serial position.

\section{Discussion}

The results of Experiment 1 replicate the results of Zentall et al. (1990) and Steirn et al. (1992). Adjusted delay errors relative to control errors were greater at PDIs 2 and 3 than they were at PDIs 1 and 4. This pattern of errors is consistent with a dual coding strategy, in which pigeons use retrospective coding at the beginning of a trial and prospective coding at the end of a trial. 


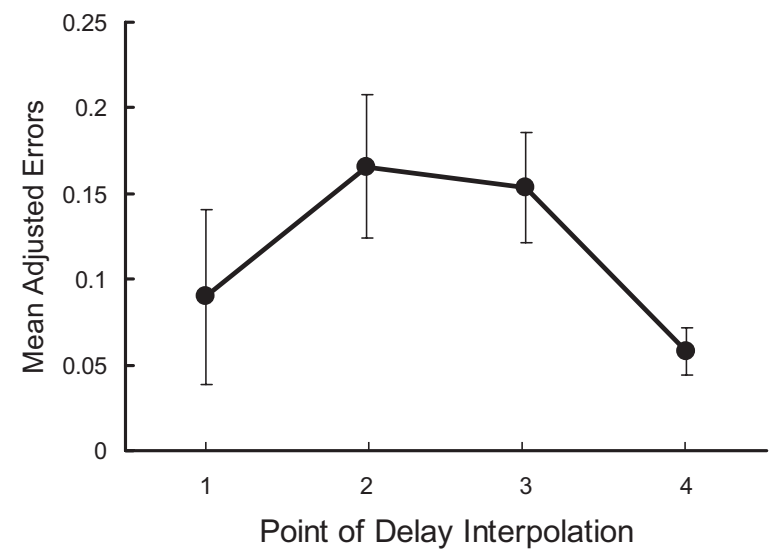

Figure 2. Mean adjusted errors attributable to the delay (delay errors control errors) in Experiment 1 (error bars represent standard error of the mean) as a function of the point of delay interpolation.

As noted earlier, in Experiment 1 a pseudodelay was used as a control procedure, in which errors on control trials were subtracted from errors on delay trials. The pseudodelay procedure was designed to correct for artifacts associated with differential choice criteria at different PDIs produced by the changing opportunity to make errors over the course of the trial. In using this subtraction method, one makes the assumption that, at each PDI, the criterion for making choices on delay trials is the same as the criterion for making choices on control trials. However, it may be that nonmemorial factors (e.g., impulsivity) affect choices more on control trials than on delay trials at a particular PDI. That is, if the pigeons are more careful in choosing after a delay than at the same point in the trial without a delay, the subtraction method may provide an inaccurate measure of errors attributable to the delay alone. Although evidence from experiments with rats does not suggest that the choice criterion on delay and control trials differs, it may be important to determine whether this assumption is also correct for pigeons.

\section{Experiment 2}

In Experiment 2, we used a testing procedure that did not require correction for opportunity to make an error created by the presence of all five keys being reilluminated after the delay by testing the pigeons with only two response keys. One of those response keys was one that had been chosen prior to the delay, and the other was one that had not yet been chosen. With this procedure, the probability of being correct by chance was $50 \%$ at all PDIs. This change in procedure allowed for the direct comparison of performance as a function of PDI.

Also included in Experiment 2 were separate control trials with no delay, on which, following a variable number of correct choices, the pigeons were given an immediate choice between a response key already chosen and another response key not yet chosen on that trial. We compared performance on delay trials with performance on these control trials at each PDI to control for all possible remaining errors not attributable to the delay.
Method

\section{Subjects}

The 6 pigeons that were used in Experiment 1 were subjects in Experiment 2.

\section{Apparatus}

The same operant chamber used in Experiment 1 was used in Experiment 2.

\section{Procedure}

After completing delay testing in Experiment 1, the pigeons were transferred to the two-alternative forced-choice procedure. In Experiment 2, all trials ended with a two-alternative forced choice between one response key already chosen on that trial and one response key not yet chosen. The two alternatives were randomly selected from among the two sets of response keys. Control sessions consisted of 12 trials each, 3 at each of the first four points in the trial following reinforcement (in this case, pseudodelays). On these trials, the pigeons were presented with the two-alternative choice following the last scheduled reinforcement, at the time that the stimulus display normally would have appeared ( 0 -s delay) The pigeons were rewarded for choosing the response key that they had not chosen earlier during that trial. Delay sessions also consisted of 12 trials each. Delay trials were similar to control trials, but they included a 30-s delay prior to the two-alternative choice. Pigeons were tested for a total of 60 sessions (40 delay sessions alternating with 20 control sessions).

\section{Results}

\section{Delay Testing}

Performance at each PDI was calculated for control and delay sessions separately. Two birds did not perform above $50 \%$ on delay trials and were excluded from the following statistical anal-

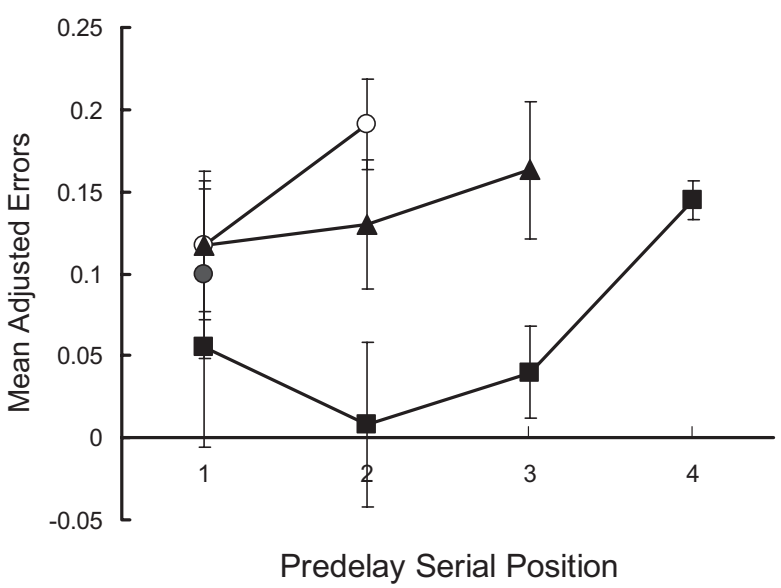

Figure 3. Mean adjusted errors attributable to the delay as a function of serial position for each point of delay interpolation (PDI) in Experiment 1 (error bars represent standard error of the mean). Filled circle $=$ PDI 1; open circles $=$ PDI 2; triangles $=$ PDI 3 ; squares $=$ PDI 4 . 
yses; however, their error patterns were very similar to those of the other birds. Errors from the remaining birds are shown in Figure 4. Errors on control sessions were, on average, lower at each PDI than errors on delay sessions. Control session errors were lower at PDIs 1 and 2 than at PDIs 3 and 4. Delay session errors were highest at PDI 1 and declined at PDIs 2, 3, and 4. We then subtracted errors on control sessions from errors on delay sessions to obtain a difference score at each PDI. The difference function, which appears in Figure 5, represents errors attributable to the delay. This function suggests that errors attributable to the delay decreased with increasing PDI.

A $t$ test revealed that performance on delay trials was above chance, with a mean percentage correct of $55.01($ SEM $=2.09)$, $t(3)=2.39, p<.05$, one-tailed. Initially, an ANOVA was conducted with the difference scores (control errors - delay errors). This test showed a significant effect of PDI, $F(3,9)=6.17, p<$ .05 , and a significant linear contrast, $F(1,3)=19.91, p<.05$, but no quadratic or cubic trend $\left(F_{\mathrm{S}}<1\right)$. Then a 2 (trial type, control vs. delay) $\times 4$ (PDI) ANOVA was conducted with the percentage error scores. The ANOVA indicated that there was a nearly significant main effect of trial type, $F(1,3)=9.35, p=.055$ (delay errors were greater than control errors), but no main effect of PDI, $F(3,9)=2.34, p>.05$. There was also a significant Trial Type $\times$ PDI interaction, $F(3,9)=6.08, p<.05$ (the difference between delay and control errors was greater at PDIs 1 and 2 than at PDIs 3 and 4). Contrasts revealed a significant linear trend for the Trial Type $\times$ PDI interaction, $F(1,3)=19.04, p<.05$, but no significant quadratic trend $(F<1)$.

To determine whether performance on delay trials varied with PDI, we performed an analysis on the data from delay trials alone. The analysis indicated that there was a significant effect of PDI, $F(3,9)=4.51, p=.03$. Furthermore, a planned trend analysis indicated that the quadratic trend was significant, $F(1,3)=10.53$, $p=.048$, but not the linear trend $(F<1)$. These analyses suggest that performance on delay trials was not flat and thus did not represent a performance floor. A similar analysis performed on the data from control trials indicated that there was no significant effect of PDI but that there was a significant linear trend, $F(1,3)=$ $31.02, p=.011$.

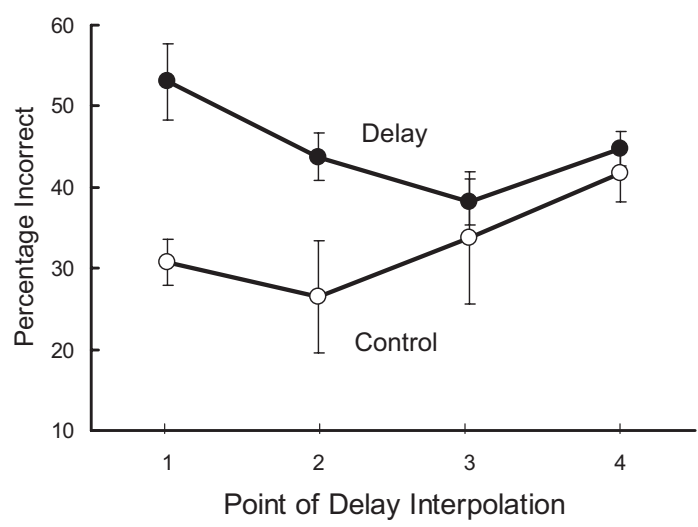

Figure 4. Percentage incorrect on control and delay trials in Experiment 2 (error bars represent standard error of the mean) as a function of the point of delay interpolation.

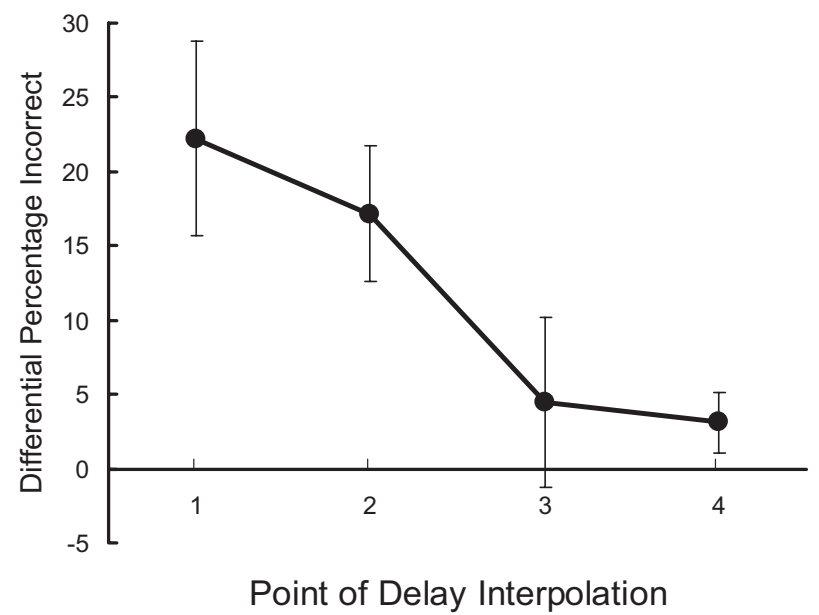

Figure 5. Percentage incorrect attributable to the delay (control performance - delay performance) in Experiment 2 (error bars represent standard error of the mean) as a function of the point of delay interpolation.

\section{Serial Position Effects}

Serial position effects were calculated and are shown in Figure 6. In Experiment 2, only one previously chosen key was presented as part of the two-alternative forced choice, and therefore only one type of error (at one serial position) could be made on each trial. Because of this difference, absolute errors were calculated at each serial position. Control trial errors were subtracted from delay trial errors at each serial position, and the difference scores were analyzed. In Experiment 2, there was no evidence of serial position effects at PDI $2, F(1,3)=0.71$; PDI $3, F(2,6)=0.47$; or PDI 4 , $F(3,9)=3.33$.

\section{Discussion}

The purpose of Experiment 2 was to more directly test for pigeons' coding strategy by avoiding having to make the assump-

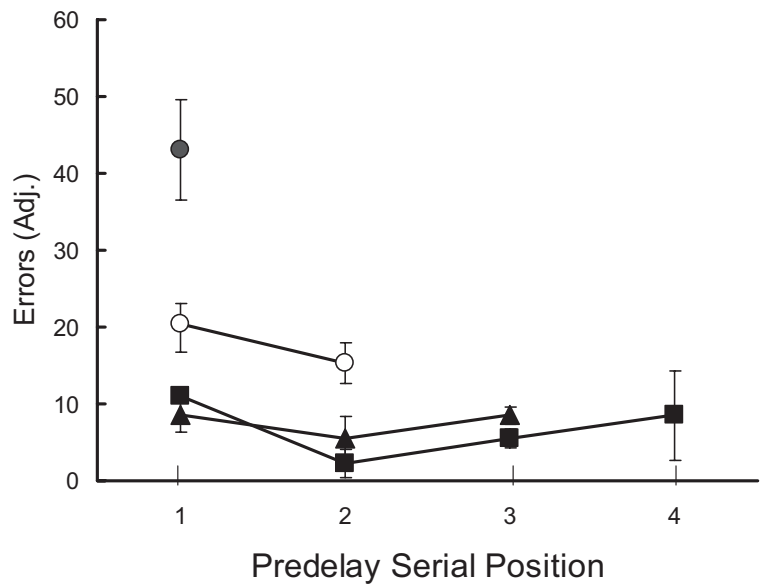

Figure 6. Number of errors attributable to the delay as a function of serial position for each point of delay interpolation (PDI) in Experiment 2 (error bars represent standard error of the mean). Adj. = adjusted; filled circle $=$ PDI 1; open circles $=$ PDI 2; triangles $=$ PDI 3; squares $=$ PDI 4. 
tion that pseudodelay control trials would control for changes in the pigeons' choice criterion resulting from the changing opportunity to make an error as the trial progressed. We accomplished this by using a two-alternative choice test to equate the probabilities of being correct by chance at all of the PDIs. We controlled for any residual errors not attributable to the delay by subtracting errors on control trials from errors on delay trials.

In Experiment 2, when errors attributable to the delay were examined, there was a monotonic decrease in errors with increasing PDI. These results are not consistent with a dual coding strategy. Instead, they are consistent with a prospective coding strategy, in which to-be-visited places are remembered throughout the trial. The later in the trial the delay occurred, the lighter was the presumed memory load and thus the fewer should be the errors attributable to the delay. The failure to find serial position effects at any PDI also supports a prospective coding hypothesis.

There are two hypotheses that can be proposed to explain the differences in the results of the two experiments. First, the pigeons in Experiments 1 and 2 might have been using different coding strategies because of the particular requirements of the two tasks. For example, in Experiment 1, the delay was followed by the reillumination of all response keys, and the pigeons were required to choose the remaining keys that they had not chosen before the delay. This task is presumably more difficult than the task used in Experiment 2, in which the delay was followed by a twoalternative forced choice. If the pigeons chose incorrectly on these two-alternative forced-choice trials, the trial ended, but in Experiment 1 , to move on to the next trial, the pigeon was forced to continue to choose (sometimes a number of already chosen keys) until it had chosen all of the response keys at least once. Because the task used in Experiment 1 was more difficult, to reduce errors, it might have encouraged the use of a dual coding strategy. In Experiment 2, the testing procedure did not require that the pigeons choose all remaining unchosen keys after the delay, and therefore a single prospective coding strategy might have been used. If the pigeons were using dual coding in Experiment 1 and were using prospective coding in Experiment 2, it would suggest that the type of coding used is quite flexible and is probably dictated by the specific demands of the task. That is, dual coding may be used when the task is more difficult.

Alternatively, in Experiment 1, early in the trial, when the probability of reinforcement by chance was $80 \%$, choosing impulsively might have been reinforced compared with choosing impulsively late in the trial, when the probability of reinforcement by chance was $20 \%$. Impulsive choice early in the trial might have occurred more often on control trials because on delay trials the delay might have disrupted an impulsive response pattern. Thus, control errors may be inflated relative to delay errors, especially early in a trial, and if these inflated control errors are subtracted from delay errors, the resulting error rate attributable to the delay would be lower than it should be. Such an artifact would produce a result consistent with a dual coding strategy and thus perhaps would mask a single prospective coding strategy.

The testing procedure used in Experiment 2 avoids the problems associated with the error adjustment required to compare error rates at the different PDIs in Experiment 1. In the two-alternative forced-choice procedure used in Experiment 2, the probability of choosing correctly by chance was always $50 \%$. Thus, no correction for opportunity was needed, and the error measure was less derived than it was in Experiment 1.

One unexpected finding in Experiment 2 was the increasing error rate on control trials as a function of increasing PDI. It may be that initially the pigeons represented the choices that they had made retrospectively. If there was a delay, however, they might have converted those choices into prospective representations of the choices they would make after the delay. The reason for the decline in accuracy on control trials as a function of PDI is that the more choices the pigeons had to remember, the more likely they were to fail to retain all of them and to treat an alternative already chosen as if it had not been chosen, resulting in an error.

\section{Experiment 3}

The error functions found in Experiment 2 (suggestive of prospective coding) were produced by the same pigeons that produced the error functions found in Experiment 1 (suggestive of dual coding). On the one hand, the fact that the same animals produced two different sets of error functions suggests that the differences cannot be attributed to individual differences among pigeons. On the other hand, it may be that the two tasks interacted in some way. That is, it is possible that the error functions found in Experiment 2 resulted from the fact that the pigeons were earlier trained with a procedure that required them to complete the trial by choosing all five keys. The purpose of Experiment 3 was to replicate the results of Experiment 2 without first requiring the pigeons to complete the trial after the delay, as in Experiment 1.

\section{Method}

\section{Subjects}

Six pigeons of the same type and maintained in the same way as those in Experiment 1 were subjects in Experiment 3.

\section{Apparatus}

The same operant chamber used in Experiment 1 was used in Experiment 3.

\section{Procedure}

Pretraining and training. Pigeons in Experiment 3 experienced the same pretraining and training phases as the pigeons in Experiment 1 .

Testing Phase 1. The testing phase was similar to the testing procedure used in Experiment 2, with alternating control and delay sessions. However, in Experiment 3 a correction procedure was used during this testing phase to encourage faster learning. After an incorrect choice, the chamber was darkened for $2.5 \mathrm{~s}$, after which the same two keys were reilluminated. This correction procedure was repeated until the pigeon chose correctly. Pigeons were reinforced for correct choices on these trials, but only the initial choice counted toward the performance measure. This procedure was in effect for 60 sessions.

Testing Phase 2. Beginning with Session 101, the correction procedure was discontinued. The noncorrection procedure was continued for 60 sessions. 
Results

\section{Training}

Pigeons made about seven errors per trial in the first block of 10 sessions. Errors declined to about three errors per trial in Block 2 and remained flat for Blocks 3 and 4 . The number of sessions to reach criterion averaged $19.5(S E M=2.64)$.

\section{Delay Testing}

The percentage of correct choices was calculated for each PDI for each pigeon for each block of 10 trials in Testing Phase 1. The mean percentage correct did not exceed $55 \%$ at any PDI, and therefore statistical analyses are not reported for these data.

One pigeon died before beginning Testing Phase 2, and therefore no data were collected for that bird. Performance at each PDI was calculated for the remaining birds on control and delay sessions and is shown in Figure 7. Errors on control sessions were generally fewer than those on delay trials. Errors on control sessions were subtracted from errors on delay sessions, and the difference function is shown in Figure 8. As in Experiment 2, the difference function suggests that errors attributable to the delay decreased with increasing PDI.

We conducted a $t$ test to determine whether performance on delay trials exceeded chance. The mean percentage correct on delay trials $(M=58.76, S E M=3.06)$ was significantly greater than chance, $t(4)=2.86, p<.05$, one-tailed. An initial ANOVA conducted with the difference scores indicated that the effect of PDI was not significant, $F(3,12)=0.76$; however, the linear trend was almost significant, $F(1,4)=6.31, p=.06$, but not the quadratic and cubic trends (both $F_{\mathrm{s}}<1$ ). A 2 (trial type) $\times 4$ (PDI) ANOVA was also conducted with percentage errors at each PDI; this indicated that there was a significant effect of trial type, $F(1,4)=53.41, p<.05$. However, neither the effect of PDI, $F(3$, $12)=2.23$, nor the PDI $\times$ Trial Type interaction was significant, $F(3,12)=0.72$. Contrasts for the Trial Type $\times$ PDI interaction revealed a nearly significant linear trend, $F(1,4)=6.04, p=.066$, but no evidence of a quadratic trend $(F<1)$.

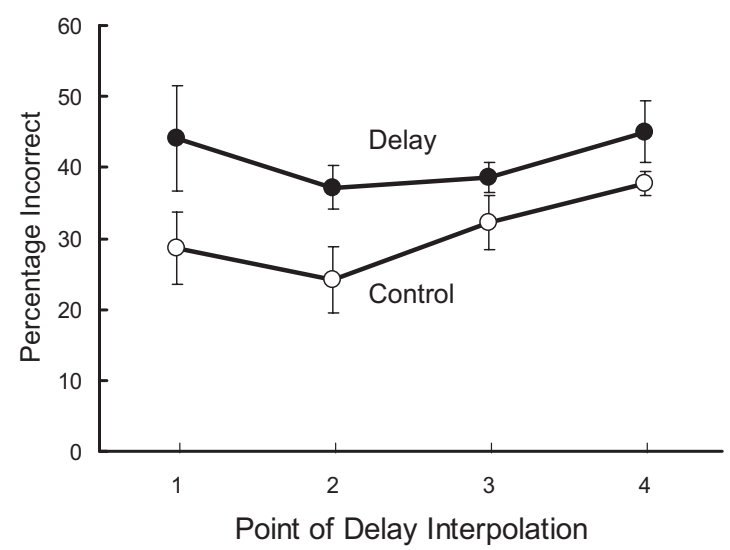

Figure 7. Percentage incorrect on control and delay trials in Experiment 3 (error bars represent standard error of the mean) as a function of the point of delay interpolation.

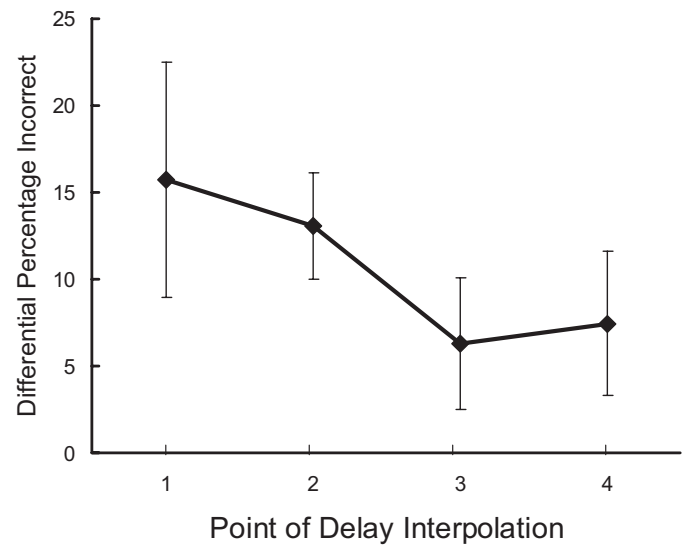

Figure 8. Percentage incorrect attributable to the delay (control performance - delay performance) in Experiment 3 (error bars represent standard error of the mean) as a function of the point of delay interpolation.

Again, to determine whether performance on delay trials varied with PDI, we performed an analysis on the data from delay trials alone. The analysis indicated that the effect of PDI was not significant, $F(3,12)<1$. A similar analysis performed on the data from control trials indicated that the effect of PDI was not significant either, $F(3,12)=2.34, p=.125$, and there were no significant trends (both $F_{\mathrm{S}}<1$ ).

\section{Serial Position Effects}

Once again, serial position effects were calculated; they are shown in Figure 9. As in Experiment 2, absolute errors were used. It should be noted that the errors shown in Figure 9 are much lower than the errors reported in Experiment 2. This difference is due to the fact that errors in Experiment 2 were calculated with more delay sessions than in Experiment 3 (40 delay sessions in Experiment 2, and 30 delay sessions in Experiment 3). Control trial

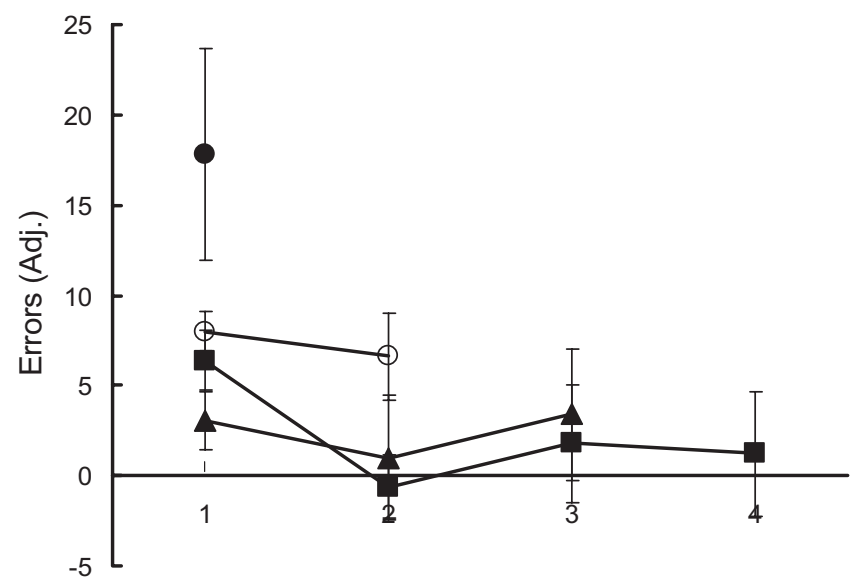

Predelay Serial Position

Figure 9. Number of errors attributable to the delay as a function of serial position for each point of delay interpolation (PDI) in Experiment 3 (error bars represent standard error of the mean). Adj. $=$ adjusted; filled circle $=$ PDI 1 ; open circles $=$ PDI 2 ; triangles $=$ PDI 3 ; squares $=$ PDI 4. 
errors were subtracted from delay trial errors, and the difference scores at each serial position were analyzed. ANOVAs indicated that there was no significant effect of serial position at PDI 2, PDI 3 , or PDI 4 (all $F \mathrm{~S}<1$ ). The absence of any serial position effects is consistent with a prospective coding strategy.

\section{Discussion}

In Experiment 3, which used the two-alternative forced-choice procedure, as in Experiment 2, pigeons performed better on control sessions than on delay sessions. As in Experiment 2, the difference in errors between control and delay sessions decreased from PDI 1 to PDI 4 . These results are consistent with the prospective coding of choices.

When compared with the results of Experiment 1, these results and those of Experiment 2 suggest that the assumption required for the error correction (i.e., that the changing opportunity to make an error affected delay trial and control trial errors equally at all PDIs) might have been responsible for the evidence for dual coding found. In Experiment 1, pigeons might have chosen with less care early in a trial, and that effect might have been even greater on control trials because there was no event that distinguished the successive choices, as there was on the two-alternative control trials in Experiments 2 and 3. In Experiment 1, it is likely that the delay allowed the pigeons to choose more carefully because the delay should have made the time of the next choice less predictable.

The procedure used in Experiments 2 and 3 allows for a more direct comparison among PDIs and also provides a distinctive test event on both control and delay trials. With this procedure, both control and delay trials included the two-alternative forced choice, a distinctive event. When this procedure was used, the results suggest that, as in Experiment 2, the pigeons were prospectively coding the response alternatives. The findings also demonstrate that the results of Experiment 2 did not depend on the delay testing used in Experiment 1 that those pigeons had had.

Although similar effects were found in Experiments 2 and 3, the magnitude of the effect found in Experiment 2 was somewhat larger that it was in Experiment 3. In Experiment 2 the largest difference between control and delay errors was about $24 \%$ and the smallest difference was about $2 \%$, so the prospective coding effect was about 22\%, whereas in Experiment 3 the largest difference between control and delay errors was about $16 \%$ and the smallest difference was about $6 \%$, so the prospective coding effect was only about $10 \%$. Comparison of Figures 4 and 6 also shows that the variability was greater in Experiment 3 than it was in Experiment 2. The best explanation for this difference is that the pigeons in Experiment 2 had had considerably more training, as they had also taken part in Experiment 1. Conversely, the similarity in the overall shape of the two functions suggests that the underlying prospective processes are similar. In support of this conclusion, a mixed-design ANOVA performed on the data from Experiments 2 and 3 with experiment, trial type, and PDI as factors indicated that the effect of trial type was statistically significant, $F(1,7)=35.35$, $p=.001$, as was the effect of PDI, $F(3,21)=4.02, p=.021$, with a significant quadratic trend, $F(1,7)=13.09, p=.009$; however, the effect of experiment was not, $F(1,7)=0.54, p=.487$. Most important, the Trial Type $\times$ PDI interaction was statistically significant, $F(3,21)=4.19, p=.018$, with a significant linear trend, $F(1,7)=25.38, p=.002$, but the Experiment $\times$ Trial Type $\times$ PDI interaction was not, $F(3,21)=0.59, p=.626$.

\section{General Discussion}

These experiments were designed to replicate and extend the results of Zentall et al. (1990) by providing a direct test of the coding processes that pigeons use when performing a radial maze analog task. Although we replicated the results of Zentall et al. (1990) and Steirn et al. (1992), the present results suggest that pigeons may use a prospective coding strategy to perform the radial maze analog task, rather than a dual coding strategy, as previously found. This novel finding in Experiment 2 was replicated in Experiment 3 with pigeons that had not been exposed to the procedures used in Experiment 1. The failure to find serial position effects in any of these experiments is consistent with the prospective coding found in Experiments 2 and 3.

However, an alternative account of the effects found in Experiments 2 and 3 may be proposed. Although choice accuracy on control trials was between $60 \%$ and $70 \%$, choice accuracy on delay trials was quite poor (between $50 \%$ and $60 \%$ at PDIs 2, 3, and 4 and only slightly above 50\% at PDI 1 in Experiment 3 and slightly below 50\% at PDI 1 in Experiment 2). Thus, one could argue that there might have been a performance floor on delay trials that prevented the delay function from showing the same increase in error rate as the control function at longer PDIs. If this account is correct, it suggests that the interaction between trial type (delay vs. control) and PDI might have been produced by a performance artifact. If the deficit due to delay is comparable at all PDIs, it suggests that the pigeons in these experiments were using neither a prospective nor a retrospective coding strategy. Furthermore, this possibility raises questions about the dual coding account proposed by Zentall et al. (1990). That is, the assumption that the pigeons' choice criterion was the same on delay and control trials at each PDI may not be accurate.

Although the above argument seems to be a parsimonious interpretation of the data, the pattern of results found in both Experiments 2 and 3 suggests that the delay functions were not flat (in both experiments, the percentage of errors on delay trials declined from PDI 1 to PDI 2 and PDI 3 and then rose again at PDI 4). Furthermore, in both experiments performance on delay trials was statistically above chance.

Given the similarity of the effects found in Experiments 2 and 3 and to further examine the effect of PDI on delay trial performance, we performed a mixed-effect ANOVA on the delay data from the two experiments. The analysis indicated that there was a significant effect of PDI, $F(3,21)=2.98, p=.05$, and a planned comparison indicated that there was also a significant quadratic trend, $F(1,7)=7.65, p=.028$. The effect of experiment was not significant, $F(1,7)=0.87$, nor was the Experiment $\times$ PDI interaction, $F(3,21)=0.81$. Thus, although the most parsimonious account of the data from Experiments 2 and 3 is that the delay produced a decrement in choice accuracy that did not vary systematically as a function of PDI, alternative accounts need to be considered.

If one considers the pigeons' performance on delay trials alone, it suggests that accuracy was best at PDIs 2 and 3. As this effect is consistent with neither a retrospective nor a prospective coding strategy, the result is difficult to interpret. Alternatively, if one 
accepts the possibility that control trial errors may vary systematically with increasing PDI, one should subtract those errors because they are not attributable to the delay. In this case, one finds a systematic decline in relative errors as a function of PDI, and this decline supports a prospective coding account. However, if pigeons do adopt such a strategy when performing this task, it is not clear what adaptive value such a strategy has. After all, it requires that the pigeons carry a relatively high memory load at the start of every trial. Perhaps further research with additional delay training and with shorter delays would result in better delay trial accuracy, making it possible to clarify the coding strategies pigeons use when performing this task.

It may also be possible to shed further light on the nature of the coding processes pigeons use when performing the radial maze analog task by isolating errors attributable to the failure to recognize choices already made from errors attributable to the failure to recognize choices yet to be made. Brown, Wheeler, and Riley (1989) attempted to make such a distinction by using a signal detection analysis on the performance of rats on the radial maze. The testing phase consisted of forced choices at various PDIs, but to better distinguish between the two kinds of errors, on test trials the authors presented the rats with one arm and a center manipulandum. On half of these test trials, the available arm had not been visited prior to the delay, and choice of this arm was reinforced. On the remaining test trials, the available maze arm had been visited prior to the delay, and choice of the center manipulandum was reinforced. If the rats were good at rejecting arms already chosen but poor at accepting arms not yet chosen, it would suggest the use of a retrospective memory code. However, if the rats were poor at rejecting arms already chosen but good at accepting arms not yet chosen, it would suggest the use of a prospective memory code. Furthermore, the degree to which each kind of error changed as a function of the PDI might suggest the use of dual coding. Finally, the degree to which the overall error rate changed with increasing PDI would suggest the degree to which there were changes in the choice criterion.

Brown et al. (1989) found results consistent with retrospective coding. They also found that rats made choices more carefully at later PDIs, a result consistent with a change in choice criterion. It may be that species and procedural differences were responsible for the difference in results between those reported by Brown et al. and those reported in this article.

Certain differences can be identified. First, rats acquire the radial maze task easily because it is consistent with their natural win-shift foraging strategy (see Hoffman et al., 1999). Rats visit each maze arm once and eat the food that is located at the end of each arm. Revisits to previously chosen arms are not reinforced because the food has already been removed. This strategy may be easy to use for rats, which naturally find small amounts of food in various places and must avoid those places visited most recently. Conversely, pigeons naturally tend to be win-stay animals, which often find larger amounts of scattered food in a particular location. Their radial maze analog task requires many sessions of training to discourage their natural tendency to revisit previously chosen keys. Perhaps this difference in natural foraging behavior makes retrospective coding more likely in rats and prospective coding more likely in pigeons. Rats are capable of using retrospective coding in the radial maze because they are less likely to revisit those alternatives. However, the use of a retrospective code by pigeons may encourage this natural tendency to revisit more than they would if they had used a prospective code.

Alternatively, the differences in coding strategy found may result from procedural differences between the experiments with rats and those with pigeons. In the experiments with rats, the rats are fed at the ends of each arm, whereas in the procedure used with pigeons, they are fed at a central feeder. When pigeons are fed at a central feeder, there is evidence that they tend to show a win-stay pattern of choice (Randall \& Zentall, 1997). However, there is also evidence that if pigeons are fed at spatially distinct locations, the acquisition of a task analogous to the present one is easier for pigeons than acquisition of the present task (see Spetch, 1990; Spetch \& Edwards, 1986; Spetch \& Honig, 1988).

Whether the present results reflect prospective coding processes by pigeons or a simpler delay trial deficit, the most important conclusion that can be drawn from the research is that the evidence for dual coding reported in earlier experiments (Steirn et al., 1992; Zentall et al., 1990) and in Experiment 1 of the present research might have resulted from the perhaps mistaken assumption that the changing criterion for making a choice, resulting from the changing probability of making an error by chance, would be the same on control and delay trials. When trials end in a two-alternative forced choice, a procedure that more directly controls for the otherwise changing probability of making an error by chance, there is no evidence of dual coding, and, in fact, the detrimental effects of the delay on choice accuracy decrease or are relatively constant as the point in the trial at which the delay is introduced increases.

\section{References}

Brown, M. F., Wheeler, E. A., \& Riley, D. A. (1989). Evidence for a shift in the choice criterion of rats in a 12-arm radial maze. Animal Learning \& Behavior, 17, 12-20.

Cook, R. G., Brown, M. F., \& Riley, D. A. (1985). Flexible memory processing by rats: Use of prospective and retrospective information in the radial maze. Journal of Experimental Psychology: Animal Behavior Processes, 11, 453-469.

Grant, D. S. (1993). Coding processes in pigeons. In T. R. Zentall (Ed.), Animal cognition: A tribute to Donald A. Riley (pp. 193-216). Hillsdale, NJ: Erlbaum.

Hoffman, C. M., Timberlake, W., Leffel, J., \& Gont, R. (1999). How is radial arm maze behavior in rats related to locomotor search tactics? Animal Learning \& Behavior, 27, 426-444.

Honig, W. K. (1978). Studies of working memory in the pigeon. In S. H. Hulse, H. Fowler, \& W. W. Honig (Eds.), Cognitive processes in animal behavior (pp. 211-248). Hillsdale, NJ: Erlbaum.

Honig, W. K. (1981). Working memory and the temporal map. In N. E. Spear \& R. R. Miller (Eds.), Information processing in animals: Memory mechanisms (pp. 167-197). Hillsdale, NJ: Erlbaum.

Honig, W. K., \& Thompson, R. K. (1982). Retrospective and prospective processing in animal working memory. Psychology of Learning and Motivation, 16, 239-283.

Kesner, R. P., \& DeSpain, M. J. (1988). Correspondence between rats and humans in the utilization of retrospective and prospective codes. Animal Learning \& Behavior, 16, 299-302.

Randall, C. K., \& Zentall, T. R. (1997). Win-stay/lose-shift and win-shift/ lose-stay learning by pigeons in the absence of overt response mediation. Behavioural Processes, 41, 227-236.

Roitblat, H. L. (1993). Representations and processes in working memory. In T. R. Zentall (Ed.), Animal cognition: A tribute to Donald A. Riley (pp. 175-192). Hillsdale, NJ: Erlbaum. 
Spetch, M. L. (1990). Further studies of pigeons' spatial working memory in the open-field task. Animal Learning \& Behavior, 18, 332-340.

Spetch, M. L., \& Edwards, C. A. (1986). Spatial memory in pigeons (Columba livia) in an open-field feeding environment. Journal of Comparative Psychology, 100, 266-278.

Spetch, M. L., \& Honig, W. K. (1988). Characteristics of pigeons' working spatial memory in an open field. Animal Learning \& Behavior, 16, 123-131.

Steirn, J. N., Zentall. T. R., \& Sherburne, L. M. (1992). Pigeons' performances of a radial-arm-maze analog task: Effect of spatial distinctiveness. Psychological Record, 42, 255-272.

Wasserman, E. A. (1986). Prospection and retrospection as processes of animal short-term memory. In D. F. Kendrick, M. E. Rilling, \& M. R. Denny (Eds.), Theories of animal memory (pp. 53-75). Hillsdale, NJ: Erlbaum.
Zentall, T. R. (1998). Symbolic representation in animals: Emergent stimulus relations in conditional discrimination learning. Animal Learning \& Behavior, 26, 363-377.

Zentall, T. R., Steirn, J. N., \& Jackson-Smith, P. (1990). Memory strategies in pigeons' performance of a radial-arm-maze analog task. Journal of Experimental Psychology: Animal Behavior Processes, 16, 358-371.

Zentall, T. R., Urcuioli, P. J., Jagielo, J. A., \& Jackson-Smith, P. (1989). Interaction of sample dimension and sample-comparison mapping on pigeons' performance of delayed conditional discriminations. Animal Learning \& Behavior, 17, 172-178.

Received December 20, 2005

Revision received October 16, 2006

Accepted October 19, 2006

\section{Members of Underrepresented Groups: Reviewers for Journal Manuscripts Wanted}

If you are interested in reviewing manuscripts for APA journals, the APA Publications and Communications Board would like to invite your participation. Manuscript reviewers are vital to the publications process. As a reviewer, you would gain valuable experience in publishing. The P\&C Board is particularly interested in encouraging members of underrepresented groups to participate more in this process.

If you are interested in reviewing manuscripts, please write to the address below. Please note the following important points:

- To be selected as a reviewer, you must have published articles in peer-reviewed journals. The experience of publishing provides a reviewer with the basis for preparing a thorough, objective review.

- To be selected, it is critical to be a regular reader of the five to six empirical journals that are most central to the area or journal for which you would like to review. Current knowledge of recently published research provides a reviewer with the knowledge base to evaluate a new submission within the context of existing research.

- To select the appropriate reviewers for each manuscript, the editor needs detailed information. Please include with your letter your vita. In the letter, please identify which APA journal(s) you are interested in, and describe your area of expertise. Be as specific as possible. For example, "social psychology" is not sufficient-you would need to specify "social cognition" or "attitude change" as well.

- Reviewing a manuscript takes time (1-4 hours per manuscript reviewed). If you are selected to review a manuscript, be prepared to invest the necessary time to evaluate the manuscript thoroughly.

Write to Journals Office, American Psychological Association, 750 First Street, NE, Washington, DC 20002-4242. 